\title{
Synthesis of novel dendritic molecules based on pyrroloanthracene units
}

\author{
Mario Smet*, David Corens and Wim Dehaen \\ Laboratory for Organic Synthesis, Department of Chemistry, University of Leuven, \\ Celestijnenlaan 200F, B-3001 Heverlee, Belgium \\ E-mail: Mario.Smet@chem.kuleuven.ac.be
}

Dedicated to Professor Georges Hoornaert on his $65^{\text {th }}$ birthday

(received 04 Dec 02; accepted 20 Dec 03; published on the web 07 Feb 03)

\begin{abstract}
Novel pyrroloanthracenes were efficiently synthesized starting from Diels-Alder adducts of anthracene. Some of these orthogonally bridged, rigid and bulky compounds were used as building blocks for the construction of monodisperse dendritic macromolecules. Molecular masses over $4 \mathrm{kDa}$ could be obtained. Further propagation was problematic because of the particular stability of the 4-(1-pyrrolyl)benzyl cation causing the instability or difficulty of formation of the analogous benzyl ether linkage in the proposed dendritic structures.
\end{abstract}

Keywords: Dendrimers, pyrroloanthracenes, Mitsunobu reaction

\section{Introduction}

During the last decade, dendrimers have attracted a lot of attention because of their unique molecular architecture. ${ }^{1}$ Numerous applications have been foreseen for these molecules e.g. as complexing agents for small molecules and indeed this concept has been experimentally proved. ${ }^{2}$ One possible strategy to increase the loading capacity of dendrimers is to incorporate bulky units in order to create large cavities in the macromolecule. The use of highly rigid building blocks will avoid collapse of the dendrimer in poor solvents. It has been demonstrated that Diels-Alder adducts of anthracene, which are highly rigid, orthogonally bridged structures, possess interesting properties to construct supramolecular entities with excellent complexing behaviour. Hydrocarbon dendrimers with triptycene monomeric units have been shown to form crystalline complexes with acetone. ${ }^{3}$ Cyclophanes consisting of Diels-Alder adducts of anthracene were found to display strong affinity towards certain tetraalkylammonium salts. ${ }^{4}$ Simple Diels-Alder adducts of anthracene were found to form clathrates with several solvents with a stoichiometry depending upon the nature of the solvent and the structure of the host. ${ }^{5}$ Fluorescent polymers in which similar entities had been incorporated were shown to be useful for the construction of sensors for dinitrotoluene which allows their use in detectors for land mines as this compound is a volatile impurity in trinitrotoluene. ${ }^{6}$ It was proved that the cavities introduced in the polymer films by the presence of the bulky anthracene adducts were essential to allow the analyte to 
penetrate in the structure and hence to induce a detectable change of the fluorescence properties of the polymers. Our group has been interested in the chemistry of anthracene for several years and we have shown that $\mathrm{N}$-phenylmaleimidoanthracenes also have remarkable inclusion properties. $^{7}$

\section{Results and Discussion}

Taking into account the described interesting properties of orthogonally bridged Diels-Alder adducts of anthracene, we wished to explore the possibility to construct dendrimers based on these species as the $\mathrm{AB}_{2}$ monomer. We chose to functionalize such adducts with ester (as protected A functionality) and phenol groups (as B functionality) allowing a deprotection strategy by reduction of the ester to alcohol and activation for coupling by well established strategies such as the Mitsunobu reaction or halogenation and subsequent Williamson ether synthesis.

In a first approach, we started from the Diels-Alder adduct 1a of anthracene and 2,5dimethoxy-2,5-dihydrofuran (2a) (Scheme 1). Although the commercial cis-trans mixture of the latter was used, only the cis adduct was isolated. Addition of $10 \mathrm{~mol} \%$ of potassium carbonate was found to increase the yield significantly, most probably by avoiding the acid catalyzed decomposition of the dihydrofuran. The obtained adduct 1a could readily be condensed in the presence of a catalytic amount of $\mathrm{p}-\mathrm{TsOH}$ with anilines bearing an electron withdrawing or neutral substituent yielding the pyrroloanthracenes 3a-h listed in scheme 1. However, the reaction was found to fail when strongly electron donating substituents are present on the aniline. This can be explained assuming the mechanism presented in scheme 2. Electron donating substituents will destabilize the supposed enamine intermediate $\mathbf{4}$ and hence disfavor the cyclization to the pyrrole. Our observation that aliphatic amines, in spite of their higher nucleophilicity, fail to react as well is consistent with this reasoning as is the observation that the yields are significantly higher when strong electron withdrawing substituents are present on the aniline. 


\section{Scheme 1}
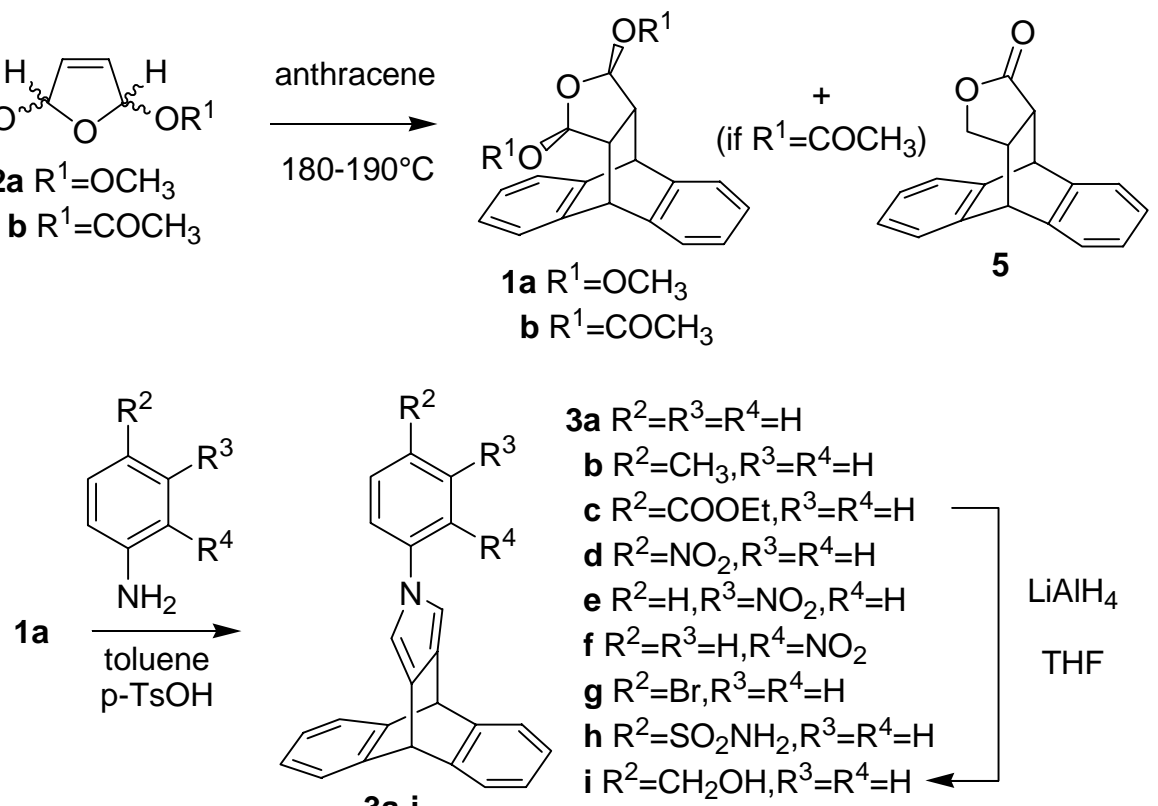

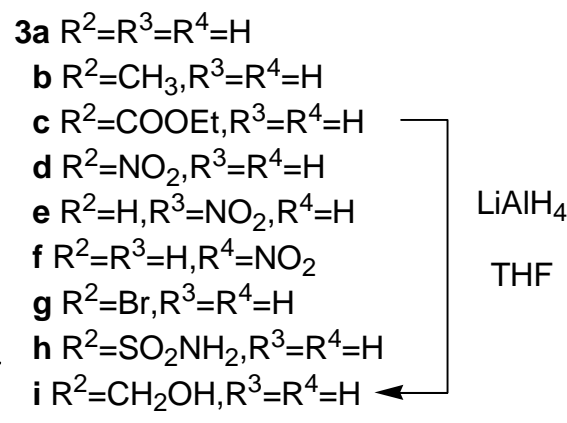

In order to extend the scope of this reaction towards anilines bearing electron donating substituents, we prepared the Diels-Alder adduct $\mathbf{1 b}$ of anthracene and 2,5-bisacetoxy-2,5dihydrofuran (2b). The latter compound was prepared via a literature procedure. ${ }^{8}$ Also in this case, addition of KOAc as a base dramatically improved the yield of the adduct although a rearrangement to lactone 5 could not be completely avoided as still $10 \%$ of this compound was formed. However, adduct $\mathbf{1 b}$ was found not to react with aromatic amines and hence this approach turned out to be useless.

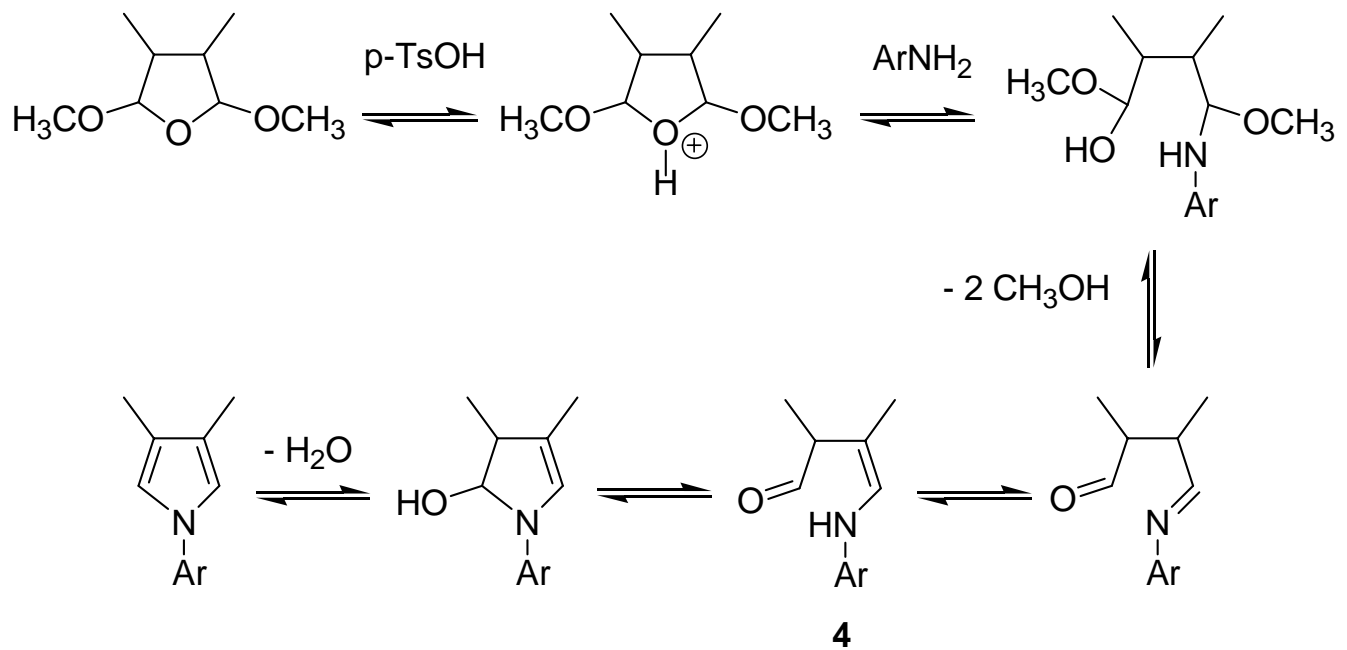

\section{Scheme 2}

The ethyl ester $3 c$ could readily be reduced with $\mathrm{LiAlH}_{4}$ yielding the benzyl alcohol $3 \mathbf{i}$ which we intended for use as peripheral unit for our dendritic branches. In order to allow easy coupling under Williamson conditions we tried to convert the alcohol function of $3 \mathbf{i}$ into a leaving group. However, numerous experiments towards this goal failed: neither the chloride (by treatment of 
the alcohol with $\mathrm{SOCl}_{2}$ ) nor the bromide (by treatment with $\mathrm{CBr}_{4} / \mathrm{PPh}_{3}$ ), nor the trifluoroacetate (by treatment with trifluoroacetic anhydride/ $\mathrm{Et}_{3} \mathrm{~N}$ ), nor the trichloroacetate (by treatment with trichloroacetyl chloride/ $\mathrm{Et}_{3} \mathrm{~N}$ ) could be detected by TLC or mass spectrometry. We found the dichloroacetate stable enough to be detected by TLC but after work up of the reaction mixture, this compound had decomposed as well. Finally, the monochloroacetate $3 \mathbf{j}$ could be obtained, characterized and substituted in a test experiment with phenolate (Scheme 3). As could be expected, this substitution did not proceed on the benzyl position but on the chloromethyl residue yielding phenoxyacetate $\mathbf{3 k}$. On the same principle, chloroacetate $3 \mathbf{j}$ could be coupled with 1,3,5tris(4-hydroxyphenyl)benzene and with 3,6-diphenyl-2,5-diketopyrrolopyrrole $\mathbf{6}^{9}$ affording the respective dendritic molecules $\mathbf{7}$ and $\mathbf{8}$ of generation 0.

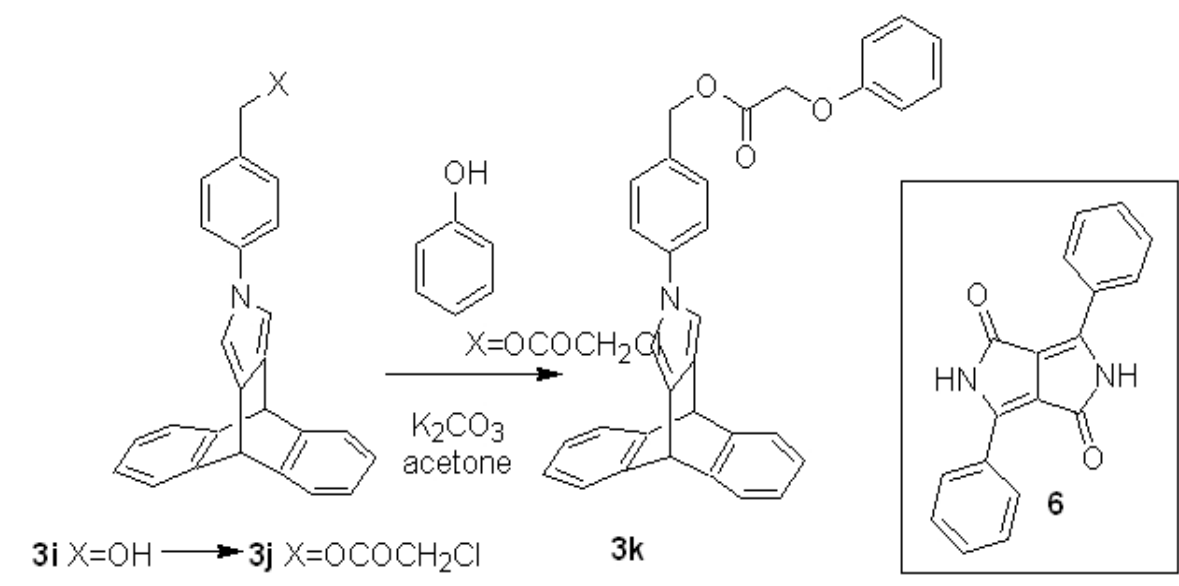

$\mathrm{CH}_{2} \mathrm{ClCOCl}, \mathrm{Et}_{3} \mathrm{~N}$
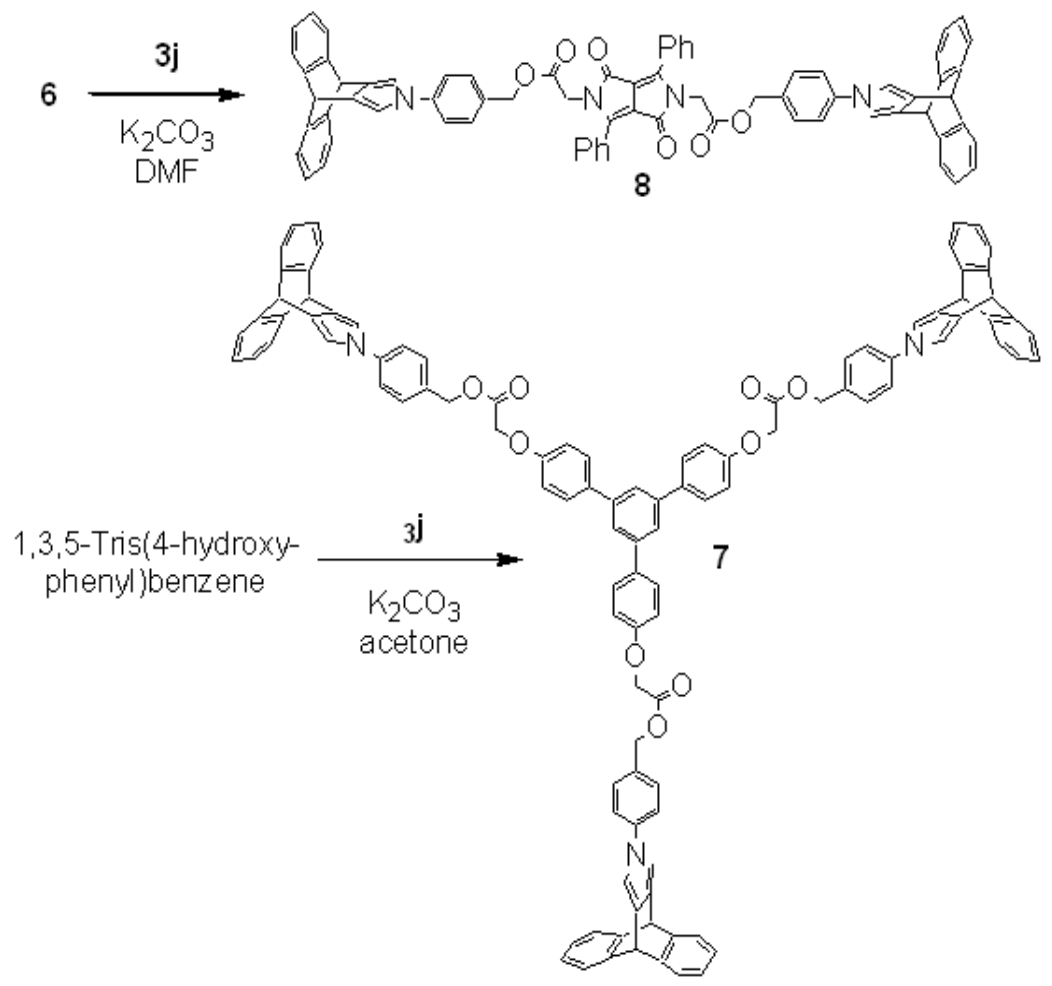

\section{Scheme 3}


In order to overcome the instability problems preventing the conversion of the alcohol function of $3 \mathbf{i}$ into a leaving group, we prepared analogous pyrroloanthracenes starting from the DielsAlder adduct 9a of anthracene and trans 1,2-dibenzoylethylene (Scheme 4). ${ }^{10}$ Although we found that this compound required more drastic conditions to be transformed into the corresponding $\mathrm{N}$ phenylpyrroloanthracenes 10a,c-e (refluxing xylene instead of toluene), this adduct also afforded the desired compounds when reacted with anilines bearing electron donating substituents. After reduction of the ester 10a to the benzyl alcohol 10b, we encountered the same problem to convert the alcohol function into a leaving group.

For the construction of a functional $\mathrm{AB}_{2}$ monomer we prepared the Diels-Alder adduct $\mathbf{9 b}$ of 1,2-bis(4-methoxybenzoyl)ethylene and anthracene. This compound could readily be transformed into the bishydroxybenzyl alcohol 10i by cyclization with ethyl 4-aminobenzoate followed by demethylation of $\mathbf{1 0 f}$ with $\mathrm{BBr}_{3}$. The latter reaction resulted to some extent in hydrolysis of the ethyl ester. However, by working at $-20^{\circ} \mathrm{C}$ this side reaction could be reduced to acceptable proportions.

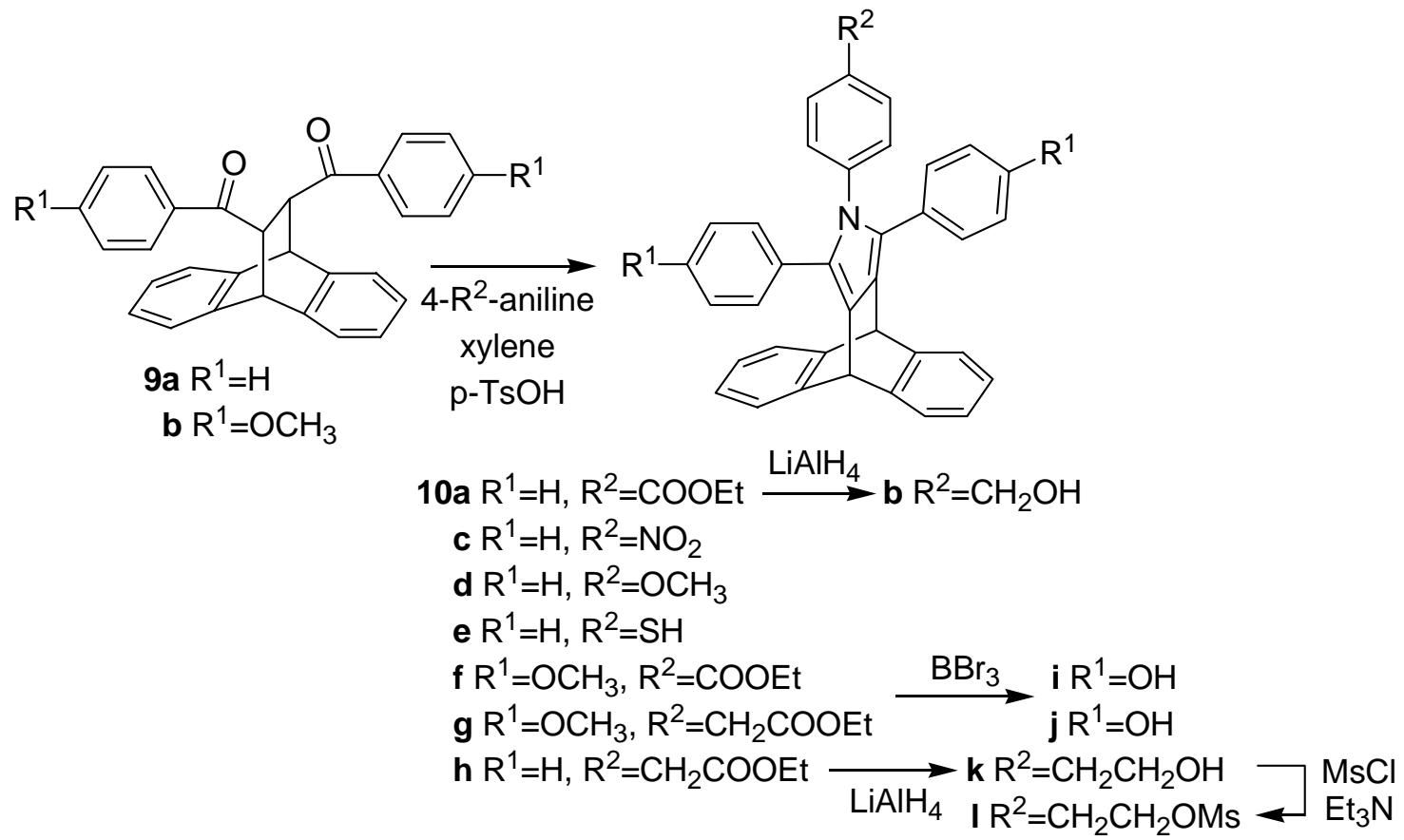

\section{Scheme 4}

To avoid Williamson ether synthesis and hence the necessity of transforming the benzylic alcohol 3i into a leaving group, we coupled $\mathrm{AB}_{2}$ monomer $\mathbf{1 0} \mathbf{i}$ with the peripheral unit $\mathbf{3 i}$ under Mitsunobu conditions (Scheme 5). The obtained G1-ester 11 was reduced to the G1-alcohol 12 with $\mathrm{LiAlH}_{4}$ and converted into the bromoacetate 13. The latter compound allowed smooth coupling with the same core reagents as cited higher yielding the G1-dendrimers 14 and 15 (Scheme 6) which could be characterized by NMR spectroscopy. ES mass spectrometry allowed to confirm the molecular mass of 2762 and 4064 Da respectively. These macromolecules were found to degrade to a large extent when kept in solution in chloroform for a few weeks. Careful analysis of the degradation products showed that the benzyl acetate groups were hydrolyzed. This is consistent with the observed instability of the 4-(pyrrol-1-yl)benzyl moiety. 

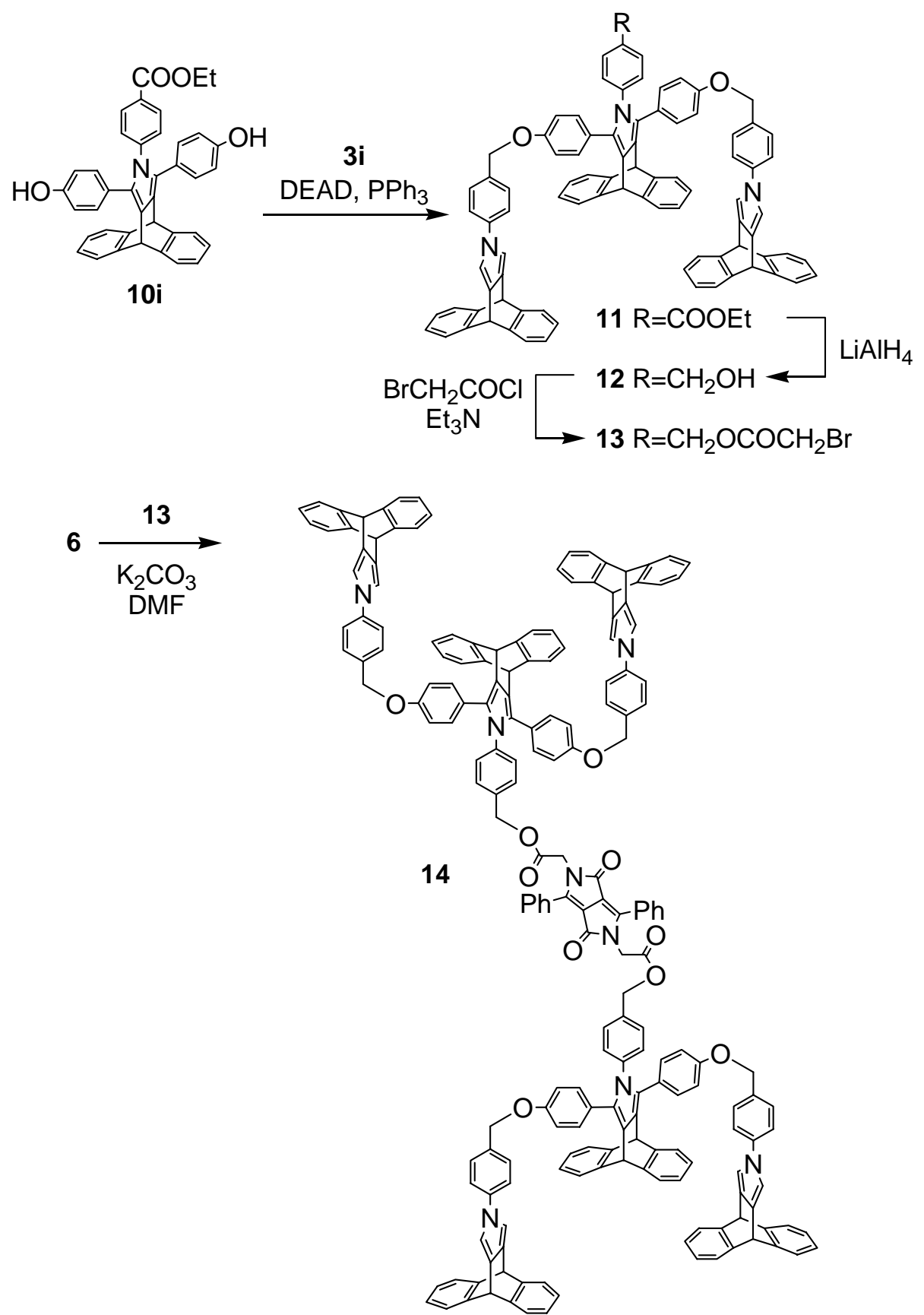

\section{Scheme 5}




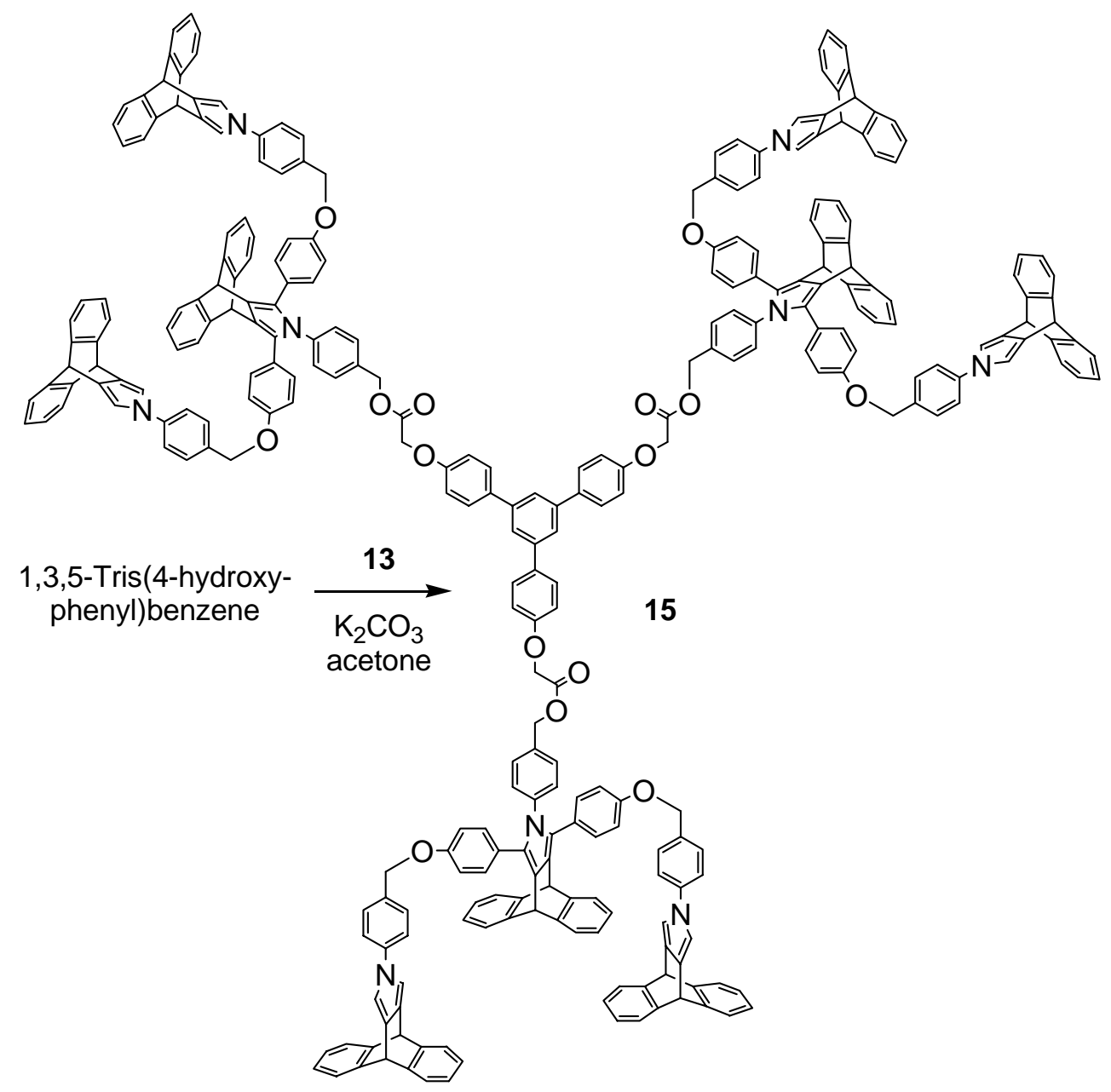

\section{Scheme 6}

Moreover, we found that even Mitsunobu coupling between G1-alcohol 12 and $\mathrm{AB}_{2}$ monomer 10i failed. Two possible side reactions were experimentally confirmed. The first one yields the hydrazine derivative 16. It is well known that products of this type can be formed upon treatment of benzyl and allyl alcohols with $\mathrm{DEAD}$ and $\mathrm{PPh}_{3}$ in the absence of a good nucleophile. ${ }^{11}$ It is evident, however, that there is no reason why our $\mathrm{AB}_{2}$ monomer $\mathbf{1 0} \mathbf{i}$ would show a lack of reactivity. Therefore, we think the extreme activation of benzyl alcohol 12 is likely to facilitate the formation of $\mathbf{1 6}$ to a notable extent. A second side reaction results in cleavage of already formed benzyl ether linkages, which is detected by the presence of alcohol $\mathbf{3 i}$ in the reaction mixture. Most probably, the benzyl ether bonds are cleaved under the influence of the intermediate formed from $\mathrm{DEAD}$ and $\mathrm{PPh}_{3}$ (Scheme 7). Again, this is a reaction for which excellent stabilization of the intermediate benzyl carbocation is a necessity. The fluorescence of the DPP core of dendrimer $\mathbf{1 4}$ was found to be almost totally quenched. This is in sharp contrast with the analogous G0-dendrimer $\mathbf{8}$ which was highly fluorescent. Most probably, backfolding of the highly electron rich 2,5-bis(4-benzyloxyphenyl)pyrrole units to the core region results in quenching of the fluorescence by electron transfer. 


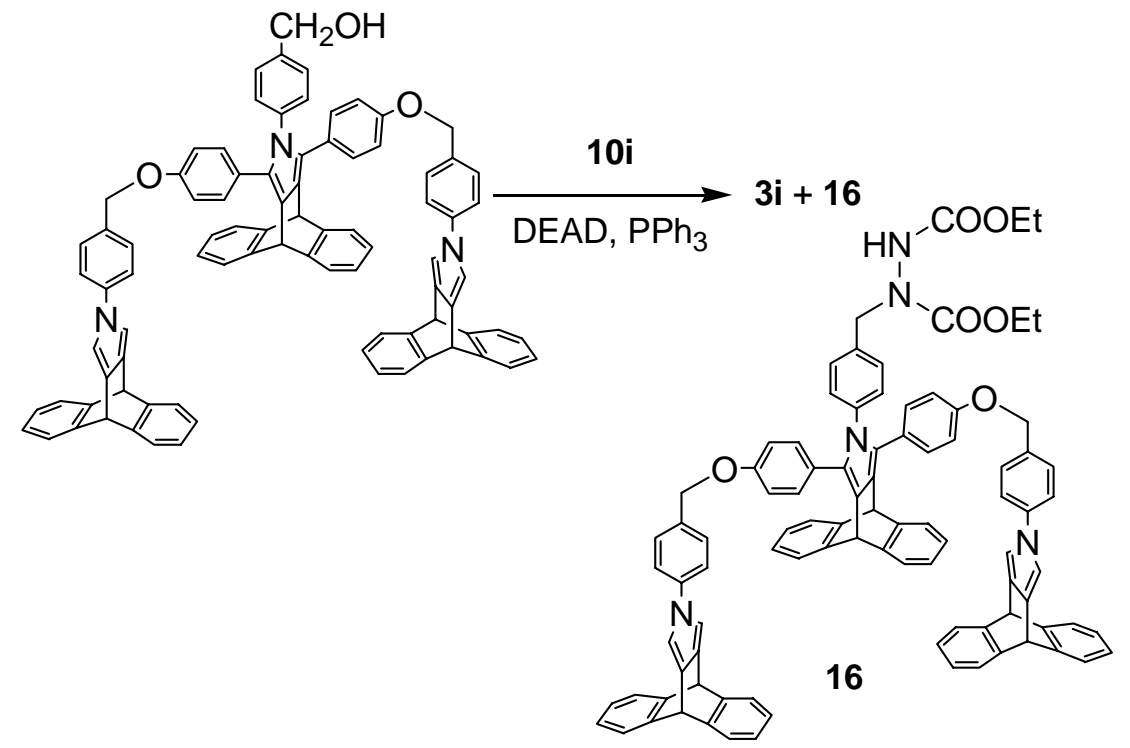

\section{Scheme 7}

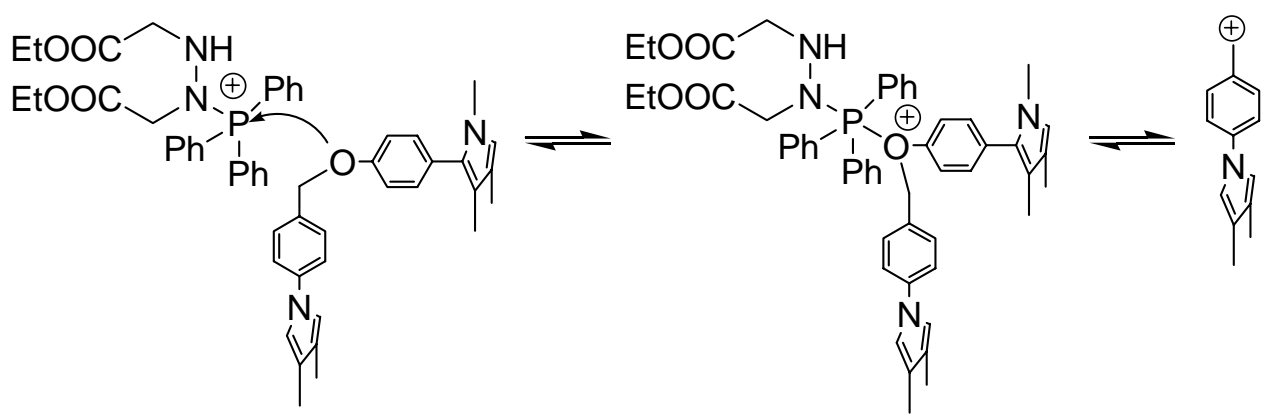

Facing these problems mentioned above, we turned our attention towards the analogous $\mathrm{AB}_{2}$ monomer 10j and peripheral unit 10k in which an extra methylene group is present (Scheme 4). The synthesis of these compounds was straightforward following the same strategy as described above. In this case, the removal of the methyl groups with $\mathrm{BBr}_{3}$ was found to be cleaner as almost no hydrolysis of the ester occurred. Conversion of alcohol 10k into mesylate 10l was unproblematic. A test experiment in which mesylate 101 was coupled with 1,3,5-tris(4hydroxyphenyl)benzene under Williamson conditions showed that, although G0-dendrimer 17 could be obtained in reasonable yield, elimination giving rise to the alkene $\mathbf{1 8}$ would be a problem for further propagation towards higher generations (Scheme 8). 


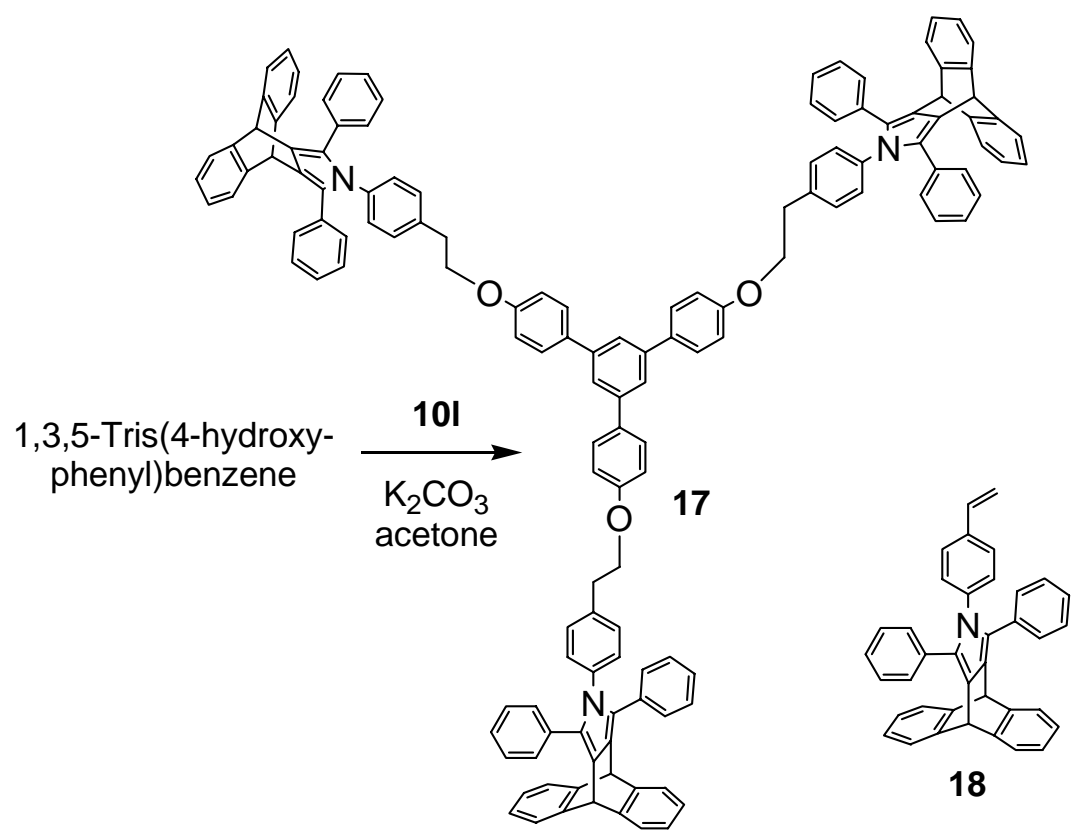

\section{Scheme 8}

Therefore, we coupled the alcohol 10k with our new $\mathrm{AB}_{2}$ monomer $\mathbf{1 0 j}$ under Mitsunobu conditions which afforded G1-dendron 19 in reasonable yield (Scheme 9). This dendron could be fully characterized by NMR spectroscopy and MS mass spectrometry ( $\mathrm{m} / \mathrm{z}=1586)$ and could be reduced cleanly with $\mathrm{LiAlH}_{4}$ affording G1-alcohol 20. However, coupling of the latter alcohol 20 with $\mathrm{AB}_{2}$ monomer $\mathbf{1 0}$ j under Mitsunobu conditions failed, as only traces of the desired G2-ester 21 could be detected by mass spectrometry.

\section{Conclusions}

We have prepared a number of new variably substituted pyrroloanthracenes by condensation of anilines with Diels-Alder adducts of anthracene. These compounds could be transformed into dendritic, monodisperse macromolecules of molecular masses exceeding $4000 \mathrm{~g} / \mathrm{mol}$. Severe problems occurred, however, upon trying to obtain higher generations, mainly caused by the very strong stabilization of the 4-(1-pyrrolyl)benzyl cation. More sophisticated coupling strategies will be necessary to overcome these problems. 

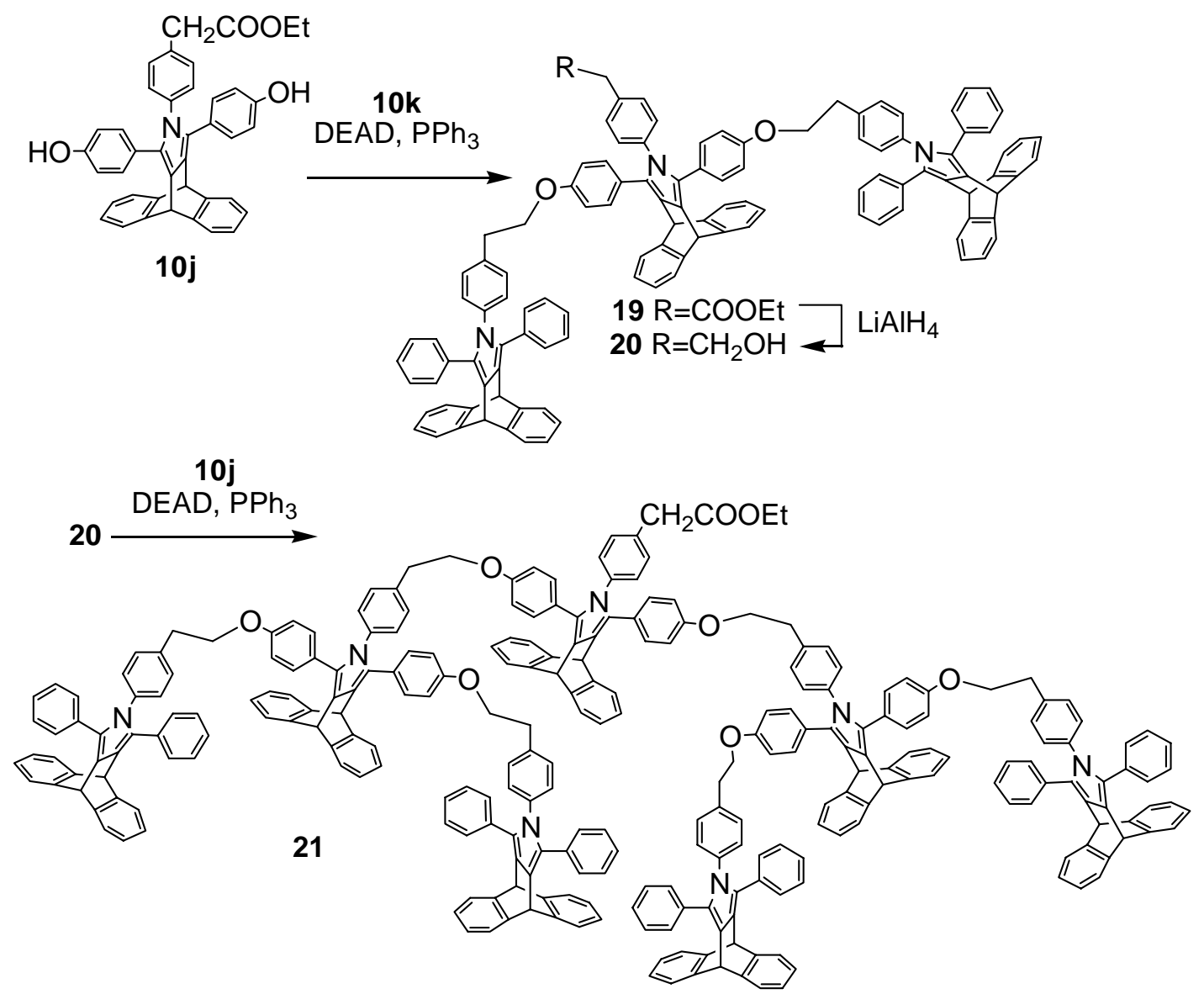

Scheme 9

\section{Experimental Section}

Synthesis of adduct 1a. A suspension of anthracene ( $14 \mathrm{~g}$; $0.078 \mathrm{~mol})$, the commerical cis-trans mixture of 2,5-dimethoxy-2,5-dihydrofuran (2a) $(10 \mathrm{~g}$; $0.078 \mathrm{~mol})$ and $\mathrm{K}_{2} \mathrm{CO}_{3}(1.1 \mathrm{~g} ; 7.8 \mathrm{mmol}$; $10 \mathrm{~mol} \%$ ) was heated for $15 \mathrm{~h}$ at $185^{\circ} \mathrm{C}$. The mixture was cooled down and the residue was crystallized from methanol yielding the adduct 1a as a white solid (22 g; 92\%): ${ }^{1} \mathrm{H}$ NMR (400 $\mathrm{MHz}, \mathrm{CDCl}_{3}$ ) $\delta$ 7.30-7.05 (3 x m, 8H), $4.63(\mathrm{~s}, 2 \mathrm{H}), 4.38(\mathrm{t}, \mathrm{J}=1.4 \mathrm{~Hz}, 2 \mathrm{H}), 3.30(\mathrm{~s}, 6 \mathrm{H}), 2.85(\mathrm{t}$, $\mathrm{J}=1.4 \mathrm{~Hz}, 2 \mathrm{H}) ;{ }^{13} \mathrm{C} \mathrm{NMR}\left(100 \mathrm{MHz}, \mathrm{CDCl}_{3}\right) \delta 142.8,140.4,126.3,126.0,124.7,123.7,109.6$, 55.2, 52.0, 46.5. MS (EI): $308\left(\mathrm{M}^{+}\right)$.

Synthesis of adduct 1b. 2,5-Diacetoxy-2,5-dihydrofuran (2b) (5.0 g; $27 \mathrm{mmol})$, anthracene (5.9 g; $33 \mathrm{mmol})$ and KOAc ( $0.5 \mathrm{~g}$ ) were heated together at $180^{\circ} \mathrm{C}$ for $15 \mathrm{~h}$ under argon atmosphere. The mixture was cooled down and dissolved in dichloromethane. Adduct $\mathbf{1 b}$ was obtained after column chromatography. $\left(\mathrm{SiO}_{2}, \mathrm{CH}_{2} \mathrm{Cl}_{2}\right.$ followed by 9:1 $\mathrm{CH}_{2} \mathrm{Cl}_{2}$-diethyl ether) as an oil (7.6 g; 80\%): ${ }^{1} \mathrm{H}$ NMR (400 MHz, $\left.\mathrm{CDCl}_{3}\right) \delta$ 7.31-7.28 (m,4H), 7.16-7.14 (m, 2H), 7.11-7.09 (m, 2H), 5.85 (s, 2H), 4.52 (s, 2H), 2.91 (s, 2H),2.02 (s, 6H). ${ }^{13} \mathrm{C}$ NMR (100 MHz, $\left.\mathrm{CDCl}_{3}\right) \delta$ 170.0, 142.4, 139.4, 126.8, 126.2, 125.0, 123.8, 102.9, 51.9, 45.74, 21.2; MS (EI): $364\left(\mathrm{M}^{+}\right)$. Lactone 5 was isolated as a white solid (0.71 g; 10\%): ${ }^{1} \mathrm{H}$ NMR $\left(400 \mathrm{MHz}, \mathrm{CDCl}_{3}\right) \delta$ 7.33-7.31 (m, 4H), 7.177.12 (m, 4H), 4.69 (d, J = 3.6 Hz, 1H), $4.30(\mathrm{t} \mathrm{J}=9.2 \mathrm{~Hz}, 1 \mathrm{H}), 4.25$ (d, J = 3.2 Hz, 1H), 3.77 (d, J 
= 4.1 Hz, 1H), $3.74(\mathrm{~d}, \mathrm{~J}=4.1 \mathrm{~Hz}, 1 \mathrm{H}), 3.16$ (d, J = 3.6 Hz), 3.13 (d, J = 3.6 Hz, 1H), 3.05-2.99 (m, 1H); ${ }^{13} \mathrm{C}$ NMR (100 MHz, $\left.\mathrm{CDCl}_{3}\right) \delta 176.1,142.3,141.6,139.9,138.7,127.0,126.8,126.4$, 126.4, 125.5, 125.1, 124.1, 123.8, 69.9, 47.5, 47.2, 45.7, 40.3; MS (EI): $262\left(\mathrm{M}^{+}\right)$.

General procedure for the synthesis of pyrroloanthracenes 3a-h. Diels-Alder adduct (0.50 g; $1.6 \mathrm{mmol})$, the appropriate amine $(2.1 \mathrm{mmol})$ and p-TsOH $(15 \mathrm{mg})$ were dissolved in toluene (40 $\mathrm{ml})$. The solution was refluxed under argon atmosphere during $12 \mathrm{~h}$. After cooling to room temperature, the solvent was evaporated and the residue purified by column chromatography ( $\mathrm{SiO}_{2}, 2: 1 \mathrm{CH}_{2} \mathrm{Cl}_{2}$-hexane).

$\boldsymbol{N}$-phenylpyrroloanthracene (3a). Obtained as an oil (65\%): ${ }^{1} \mathrm{H}$ NMR (400 $\left.\mathrm{MHz}, \mathrm{CDCl}_{3}\right) \delta$ 7.33 (m, 4H), $7.26(\mathrm{dt}, 2 \mathrm{H}), 7.17(\mathrm{dt}, 2 \mathrm{H}), 7.07$ (tt, 1H), $6.971 \mathrm{H}), 6.79(\mathrm{~m}, 4 \mathrm{H}), 6.85(\mathrm{~s}, 2 \mathrm{H})$, 5.32 (s, 2H); ${ }^{13} \mathrm{C}$ NMR $\left(100 \mathrm{MHz}, \mathrm{CDCl}_{3}\right) \delta 146.6,141.1,132.8,129.3,125.0,124.6,123.4$, 119.9, 111.1, 47.2; MS (EI): $319\left(\mathrm{M}^{+}\right)$.

$\boldsymbol{N}$-(4-methylphenyl)pyrroloanthracene (3b). Obtained as an oil (58 \%): ${ }^{1} \mathrm{H}$ NMR (400 MHz, $\left.\mathrm{CDCl}_{3}\right) \delta$ 7.30-7.35 (m, 4H), 7.09 (s, 4H), 6.79 (m, 4H), 6.84 (s, 2H), 5.32 (s, 2H), 2.29 (s, 3);

${ }^{13} \mathrm{C}$ NMR $\left(100 \mathrm{MHz}, \mathrm{CDCl}_{3}\right) \delta 146.7,138.8,134.2,132.5,129.8,125.0,123.3,119.9,111.2$, 47.2, 20.7; MS (EI): $333\left(\mathrm{M}^{+}\right)$.

$\mathbf{N}$-(4-ethoxycarbonylphenyl)pyrroloanthracene (3c). Obtained as an amorphous solid after column chromatography $\left(\mathrm{SiO}_{2}, \mathrm{CH}_{2} \mathrm{Cl}_{2}\right)(86 \%)$ : ${ }^{1} \mathrm{H}$ NMR $\left(400 \mathrm{MHz}, \mathrm{CDCl}_{3}\right) \delta 7.99(\mathrm{~d}, 2 \mathrm{H})$, 7.30-7.38 (m, 4H), 7.22 (d, 2H), 6.96-7.02 (m, 4H), 6.92 (s, 2H), 5.32 (s, 2H), 4.33 (q, 2H), 1.35 $(\mathrm{t}, 3 \mathrm{H}) ;{ }^{13} \mathrm{C}$ NMR $\left(100 \mathrm{MHz}, \mathrm{CDCl}_{3}\right) \delta 166.0,146.1,144.0,133.8,131.1,126.1,125.2,123.4$, 118.4, 110.7, 60.8, 47.0, 14.3; MS (EI): $391\left(\mathrm{M}^{+}\right)$.

$\mathbf{N}$-(4-nitrophenyl)pyrroloanthracene (3d). Obtained as a yellow amorphous solid (85\%): ${ }^{1} \mathrm{H}$ NMR (400 MHz, CDCl $)_{3} \delta 8.19$ (d, 2H), 7.32-7.40 (m, 4H), 7.27 (d, 2H), 6.99-7.17 (m, 4H), 6.97 (s, 2H), 5.37 (s, 2H); ${ }^{13} \mathrm{C}$ NMR (100 MHz, $\left.\mathrm{CDCl}_{3}\right) \delta 145.8,145.7,143.8,134.8,125.5$, 125.4, 123.5, 118.3, 110.7, 46.9; MS (EI): $364\left(\mathrm{M}^{+}\right)$.

$\boldsymbol{N}$-(3-nitrophenyl)pyrroloanthracene (3e). Obtained as a yellow amorphous solid (76\%): ${ }^{1} \mathrm{H}$ NMR (400 MHz, $\left.\mathrm{CDCl}_{3}\right) \delta 8.06\left(\mathrm{t}, \mathrm{J}_{\mathrm{m}}=2.5 \mathrm{~Hz}, 1 \mathrm{H}\right), 7.93\left(\mathrm{dt}, \mathrm{J}_{\mathrm{o}}=10.5 \mathrm{~Hz}, \mathrm{~J}_{\mathrm{m}}=2.5 \mathrm{~Hz}, 1 \mathrm{H}\right)$, $7.52\left(\mathrm{dt}, \mathrm{J}_{\mathrm{o}}=10.5 \mathrm{~Hz}, \mathrm{~J}_{\mathrm{m}}=2.5 \mathrm{~Hz}, 1 \mathrm{H}\right), 7.46\left(\mathrm{t}, \mathrm{J}_{\mathrm{o}} 10.5 \mathrm{~Hz}, 1 \mathrm{H}\right), 7.34-7.39$ (m, 4H), 6.98-7.05 (m, 4H), 6.94 (s, 2H), 5.35 (s, 2H); ${ }^{13} \mathrm{C}$ NMR (100 MHz, $\left.\mathrm{CDCl}_{3}\right) \delta$ 149.0, 146.0, 141.9, 134.3, 130.3, 125.3, 124.7, 123.5, 118.9, 114.0, 110.8, 46.9; MS (EI): $364\left(\mathrm{M}^{+}\right)$.

$\boldsymbol{N}$-(2-nitrophenyl)pyrroloanthracene (3f). Obtained as a yellow amorphous solid (65\%): ${ }^{1} \mathrm{H}$ $\operatorname{NMR}\left(400 \mathrm{MHz}, \mathrm{CDCl}_{3}\right) \delta 7.71\left(\mathrm{dd}, \mathrm{J}_{\mathrm{o}}=10.5 \mathrm{~Hz}, \mathrm{~J}_{\mathrm{m}}=2.5 \mathrm{~Hz}, 1 \mathrm{H}\right), 7.52\left(\mathrm{td}, \mathrm{J}_{\mathrm{o}}=10.5 \mathrm{~Hz}, \mathrm{~J}_{\mathrm{m}}=\right.$ $2.5 \mathrm{~Hz}, 1 \mathrm{H}), 7.28-7.37$ (m, 6H), 6.96-7.05 (m, 4H), 6.57 (s, 2H), $5.29 \mathrm{~s}, 2 \mathrm{H})$; ${ }^{13} \mathrm{C}$ NMR (100 $\left.\mathrm{MHz}, \mathrm{CDCl}_{3}\right) \delta 146.3,144.6,134.3,133.6,132.9,127.3,126.2,125.1,124.8,123.5,112.8,47.0$; MS (EI): $364\left(\mathrm{M}^{+}\right)$.

$\mathbf{N}$-(4-bromophenyl)pyrroloanthracene (3g). Obtained as a white amorphous solid (46\%): ${ }^{1} \mathrm{H}$ NMR (400 MHz, $\left.\mathrm{CDCl}_{3}\right) \delta 7.38(\mathrm{~d}, 2 \mathrm{H}), 7.03$ (d, 2H), 6.94-7.00 (m, 4H), 6.79 (s, 2H), 5.30 (s, 2H); ${ }^{13} \mathrm{C}$ NMR (100 MHz, $\left.\mathrm{CDCl}_{3}\right) \delta$ 146.4, 140.1, 133.3, 125.1, 123.4, 121.2 (2C), 117.4, 110.9, 47.1; MS (EI): 399-397 $\left(\mathrm{M}^{+}\right)$.

$\mathbf{N}$-(4-sulfamidophenyl)pyrroloanthracene (3h). Obtained as a white amorphous solid (62\%): ${ }^{1} \mathrm{H}$ NMR (400 MHz, $\mathrm{CDCl}_{3}$ ) $\delta 7.77$ (d, 2H), 7.62 (d, 2H), 7.35-7.42 (m, 4H), 7.31 (s, 2H), 7.29 (s, 2H, $\mathrm{NH}_{2}$ ), 6.94-7.03 (m, 4H), 5.45 (s, 2H); ${ }^{13} \mathrm{C}$ NMR (100 MHz, $\left.\mathrm{CDCl}_{3}\right) \delta$ 146.2, 142.7, 139.3, 133.3, 127.3, 124.9, 123.4, 118.3, 110.9, 45.8; MS (EI): $398\left(\mathrm{M}^{+}\right)$. 
G0-OH 3i. Ethyl ester 3c (4.7 g; $12 \mathrm{mmol}$ ) was dissolved in dry THF (50 ml). This solution was added in a dropwise manner to a suspension of $\mathrm{LiAlH}_{4}(0.5 \mathrm{~g} ; 14 \mathrm{mmol})$ in dry THF (10 ml) in an ice bath. After complete addition the solution was refluxed for $1 \mathrm{~h}$. After cooling to room temperature, an aqueous solution of $\mathrm{NaOH}(1 \mathrm{M})$ was added dropwise (approx. $20 \mathrm{ml}$ ). The precipitate was filtered and washed with THF. The solution was washed with $\mathrm{MgSO}_{4}$ and evaporated. After column chromatography $\mathrm{SiO}_{2} 3: 1 \mathrm{CH}_{2} \mathrm{Cl}_{2}-\mathrm{Et}_{2} \mathrm{O}$ ), the alcohol $3 \mathbf{i}$ was obtained as a foam (3.6 g; 86\%): ${ }^{1} \mathrm{H}$ NMR (400 MHz, $\left.\mathrm{CDCl}_{3}\right) \delta 7.30-7.38(\mathrm{~m}, 4 \mathrm{H}), 7.26(\mathrm{~d}, 2 \mathrm{H}), 7.17(\mathrm{~d}$, 2H), 6.95-7.02 (m, 4H), 6.86 (s, 2H), 5.32 (s, 2H), 4.56 (s, 2H), $1.72(\mathrm{~s}, 1 \mathrm{H}) ;{ }^{13} \mathrm{C}$ NMR (100 $\left.\mathrm{MHz}, \mathrm{CDCl}_{3}\right) \delta 146.5,140.5,137.0,132.8,128.1,125.1,123.3,119.8,111.0,64.7,47.1$; MS (EI): $349\left(\mathrm{M}^{+}\right)$.

Chloroacetate 3j. The alcohol $3 \mathbf{i}(1.2 \mathrm{~g} ; 3.5 \mathrm{mmol})$ and $\mathrm{Et}_{3} \mathrm{~N}$ (0.70 g; $\left.6.9 \mathrm{mmol}\right)$ were dissolved in $\mathrm{CH}_{2} \mathrm{Cl}_{2}(20 \mathrm{ml})$ and the solution was cooled to $-10^{\circ} \mathrm{C}$. Chloroacetyl chloride $(0.78 \mathrm{~g} ; 6.9$ mmol) was added and the mixture was stirred for $30 \mathrm{~min}$ at room temperature. The reaction mixture was quenched with water and the organic layer was separated, dried over $\mathrm{MgSO}_{4}$ and evaporated. Chloroacetate $\mathbf{3 j}$ was obtained after column chromatography $\left(\mathrm{SiO}_{2}, \mathrm{CH}_{2} \mathrm{Cl}_{2}\right)$ as a colorless oil (1.2 g; 81\%): ${ }^{1} \mathrm{H}$ NMR (250 MHz, $\left.\mathrm{CDCl}_{3}\right) \delta$ 7.36-7.34 (m, 4H), 7.31 (d, J = 8.2 Hz, 2H), 7.19 (d, J = 8.2 Hz, 2H), 7.01-6.95 (m, 4H), 6.88 (s, 2H), 5.33 (s, 2H), 5.13 (s, 2H), 4.07 (s, 2H); MS (EI) $425\left(\mathrm{M}^{+}\right)$.

G0-dendrimer 7. Chloroacetate $3 \mathbf{j}$ (0.52 g; $1.2 \mathrm{mmol})$, 1,3,5-tris(4-hydroxyphenyl)benzene (0.11 g; $0.31 \mathrm{mmol})$ and $\mathrm{K}_{2} \mathrm{CO}_{3}(17 \mathrm{mg} ; 1.2 \mathrm{mmol})$ were suspended in acetone and the suspension was refluxed during 48 hours under argon atmosphere. After evaporation of the reaction mixture and column chromatography $\left(\mathrm{SiO}_{2}, \mathrm{CH}_{2} \mathrm{Cl}_{2}\right) \mathrm{G0}$-dendrimer 7 was obtained as glassy solid (0.32 g; 68\%: ${ }^{1} \mathrm{H}$ NMR (250 MHz, $\left.\mathrm{CDCl}_{3}\right) \delta 7.63(\mathrm{~s}, 3 \mathrm{H}), 7.58$ (d, J = 8.2 Hz, 6H), 7.35-7.30 (m,12 H), 7.28 (d, J = 8.2 Hz, 6H), 7.16 (d, J = 8.2 Hz, 6H), 7.01-6.93 (m, 18H), 6.83 (s, 6H), 5.31 (s, 6H), 5.19 (s, 6H), 4.69 (s, 6H) MS (ES) m/z: $1523\left(\mathrm{MH}^{+}\right)$.

G0-dendrimer 8. DPP 6 (0.17 g; $0.59 \mathrm{mmol}$ ), chloroacetate $3 \mathbf{j}(0.75$ g, $1.8 \mathrm{mmol})$ and $\mathrm{K}_{2} \mathrm{CO}_{3}$ ( $0.25 \mathrm{~g} ; 1.8 \mathrm{mmol})$ were suspended in DMF $(20 \mathrm{ml})$ and the mixture was heated at $80^{\circ} \mathrm{C}$ during 48 hours. After cooling to room temperature, evaporation of the solvent and column chromatography $\left(\mathrm{SiO}_{2}, \mathrm{CH}_{2} \mathrm{Cl}_{2}\right) \mathrm{G} 0$-dendrimer $\mathbf{8}$ was obtained as a yellow fluorescing oil $(0.47$ g; 73\%): ${ }^{1} \mathrm{H}$ NMR $\left(250 \mathrm{MHz}, \mathrm{CDCl}_{3}\right) \delta$ 7.68-7.63 (m, 4H), 7.43-7.32 (m, 10H), 7.23-7.18 (m, 8H), 7.01-6.97 (m, 8H), 6.87 (s, 4H), 5.33 (s, 4H), 5.11 (s, 2H), 4.51 (s, 4H); MS (ES) m/z: 1067 $\left(\mathrm{MH}^{+}\right)$.

General procedure for the preparation of triphenylpyrroloanthracenes 10a,c-e. Diels-Alder adduct 9a (1.0 g; $2.4 \mathrm{mmol})$, the appropriate amine (0.44 g; $2.7 \mathrm{mmol})$ and p-TsOH (15 mg) were dissolved in m-xylene $(40 \mathrm{ml})$ and refluxed under argon atmosphere during $48 \mathrm{~h}$ under azeotropic removal of water. After cooling to room temperature the residue was crystallized from methanol (20 ml).

$\mathbf{N}$-(4-ethoxycarbonylphenyl)diphenylpyrroloanthracene (10a) was obtained as a white solid (1.1 g; 83\%): $\mathrm{Mp}>300^{\circ} \mathrm{C} .{ }^{1} \mathrm{H}$ NMR $\left(250 \mathrm{MHz}, \mathrm{CDCl}_{3}\right) \delta 7.76$ (d, J = 8.0 Hz, 2H), 7.38-7.32 (m, 4H), 7.25-7.20 (m, 6H), 7.06-6.95 (m, 6H), 6.79 (d, J = 8.0 Hz, 2H), 5.39 (s, 2H), 4.28 (q, J = 6.7 $\mathrm{Hz}, 2 \mathrm{H}), 1.33$ (t, J = 6.7 Hz, 3H); MS (EI) $543\left(\mathrm{M}^{+}\right)$.

$\mathbf{N}$-(4-nitrophenyl)diphenylpyrroloanthracene (10c) was obtained as a yellow solid (78\%): ${ }^{1} \mathrm{H}$ NMR (250 MHz, $\left.\mathrm{CDCl}_{3}\right) \delta 7.93(\mathrm{~d}, \mathrm{~J}=8.8 \mathrm{~Hz}, 2 \mathrm{H}), 7.41-7.36$ (m, 4H), 7.29-7.24 (m, 6H), 7.056.99 (m, 6H), 6.84 (d, J = 8.8 Hz, 2H), 5.39 (s, 2H); MS (EI) $516\left(\mathrm{M}^{+}\right)$. 
$\boldsymbol{N}$-(4-methoxyphenyl)diphenylpyrroloanthracene (10d) was obtained as a white solid (73\%): ${ }^{1} \mathrm{H}$ NMR (250 MHz, $\mathrm{CDCl}_{3}$ ) $\delta$ 7.41-7.36 (m, 4H), 7.29-7.24 (m, 6H), 7.05-6.99 (m, 6H), 6.71 (d, $\mathrm{J}=8.4 \mathrm{~Hz}, 2 \mathrm{H}), 6.57$ (d, J = 8.4 Hz, 2H), 5.39 (s, 2H), 3.67 (s, 3H); MS (EI) $501\left(\mathrm{M}^{+}\right.$).

$\mathrm{N}$-(4-sulfanylphenyl)diphenylpyrroloanthracene (10e) was obtained as a white solid (78\%): ${ }^{1} \mathrm{H}$ NMR (250 MHz, $\mathrm{CDCl}_{3}$ ) $\delta$ 7.41-7.36 (m, 4H), 7.29-7.24 (m, 6H), 7.04-6.97 (m, 10H), 6.62 (d, J = 8.4 Hz, 2H), 5.39 (s, 2H), 3.37 (s, 1H); MS (EI) $503\left(\mathrm{M}^{+}\right)$.

Benzyl alcohol 10b. The ester 10a $(1.0 \mathrm{~g} ; 1.7 \mathrm{mmol})$ was dissolved in dry THF (5 ml) and was added in a dropwise manner to a suspension of $\mathrm{LiAlH}_{4}(0.13 \mathrm{~g} ; 3.3 \mathrm{mmol})$ in THF (5 ml). After complete addition, the solution was refluxed for $30 \mathrm{~min}$. After cooling down to room temperature, a solution of $\mathrm{NaOH}(1 \mathrm{M})$ was added (approx. 3ml) and the suspension was filtered and the precipitate washed with THF. The organic layer was dried over $\mathrm{MgSO}_{4}$ and evaporated. The alcohol 10b was obtained after column chromatography $\left(\mathrm{SiO}_{2}, \mathrm{CH}_{2} \mathrm{Cl}_{2}\right)$ as a foam $(0.85 \mathrm{~g}$; 89\%): ${ }^{1} \mathrm{H}$ NMR (250 MHz, $\mathrm{CDCl}_{3}$ ) $\delta$ 7.39-7.33 (m, 4H), 7.24-7.18 (m, 6H), 7.06-6.98 (m, 8H), 6.78 (d, J = 8.5 Hz, 2H, 5.39 (s, 2H), 4.57 (d, br, 2H); MS (EI) $501\left(\mathrm{M}^{+}\right)$.

11,12-Bis(4-methoxyphenylcarbonyl)-9,10-ethanoanthracene (9b). Bis-1,2-(4methoxybenzoyl)ethylene (10 g; $34 \mathrm{mmol})$ and anthracene $(6.0 \mathrm{~g} ; 34 \mathrm{mmol})$ were heated together at $80^{\circ} \mathrm{C}$ during $1 \mathrm{~h}$. The reaction mixture was cooled down to $50^{\circ} \mathrm{C}$ and ethanol $(120 \mathrm{ml})$ was added. After vigorous stirring, the precipitate was filtered off, washed with methanol (3 x 20 $\mathrm{ml}$ ) and dried in vacuum which yielded $9 \mathrm{~b}$ as a white solid (14 g; 87\%): ${ }^{1} \mathrm{H}$ NMR (250 $\mathrm{MHz}$, $\left.\mathrm{CDCl}_{3}\right) \delta 7.94(\mathrm{~d}, \mathrm{~J}=8.5 \mathrm{~Hz}, 4 \mathrm{H}), 7.38$ (d, J = 7 Hz, 2H), 7.17 (td, J = 7 Hz, J = 2 Hz, 2H), 7.08 (td, J = $7 \mathrm{~Hz}, \mathrm{~J}=2 \mathrm{~Hz}, 2 \mathrm{H}), 7.01$ (d, J = $7 \mathrm{~Hz}, 2 \mathrm{H}), 6.92$ (d, J = 8.5 Hz, 4H), 4.60 (s, 2H), 4.49 (s, 2H), 3.88 (s, 6H); MS (EI) m/z: $474\left(\mathrm{M}^{+}\right)$.

Ethyl ester 10f. Diels-Alder adduct 9b $(0.50 ; 1.1 \mathrm{mmol})$, ethyl 4-aminobenzoate (0.26 g; 1.6 $\mathrm{mmol})$ and p-TsOH $(0.1 \mathrm{~g})$ were dissolved in m-xylene $(40 \mathrm{ml})$ and the mixture was heated at reflux temperature in a Dean-Stark tube during $24 \mathrm{~h}$. After cooling to room temperature and evaporation of the solvent, methanol $(20 \mathrm{ml})$ was added. After filtration and washing with methanol (3 x $5 \mathrm{ml}$ ), pyrroloanthracene 10f was obtained as a white solid $(0.57 \mathrm{~g} ; 84 \%):{ }^{1} \mathrm{H}$ NMR (250 MHz, CDCl $)_{3} \delta 7.76(\mathrm{~d}, \mathrm{~J}=8.5 \mathrm{~Hz}, 2 \mathrm{H}), 7.39-7.33(\mathrm{~m}, 4 \mathrm{H}), 7.03-6.98(\mathrm{~m}, 4 \mathrm{H}), 6.92$ (d, J = 8 Hz, 4H), 6.79-6.73 (2 x d, 6H), 5.37 (s, 2H), 4.29 (q, J = 7 Hz, 2H), 3.81 (s, 6H), 1.33 (t, $\mathrm{J}=7 \mathrm{~Hz}, 3 \mathrm{H})$; MS (EI) m/z: $603\left(\mathrm{M}^{+}\right)$.

Diphenol 10i. Dimethoxy derivative 10f $(0.20$; $0.33 \mathrm{mmol})$ was dissolved in dichloromethane $(20 \mathrm{ml})$ and the solution was placed under argon and cooled to $-78^{\circ} \mathrm{C}$. Via a septum, $\mathrm{BBr}_{3}(0.33$ g; $1.3 \mathrm{mmol}$ ) was added and the mixture was kept at $-18^{\circ} \mathrm{C}$ during $15 \mathrm{~h}$. Water $(5 \mathrm{ml})$ and ethyl acetate $(20 \mathrm{ml})$ were added and the water layer was extracted once more with ethyl acetate (20 $\mathrm{ml})$. The combined organic layers were dried over $\mathrm{MgSO}_{4}$ and evaporated in vacuo. Diphenol $10 \mathbf{i}$ was obtained as a light brown powder $(0.14 \mathrm{~g} ; 75 \%)$ after column chromatography $\left(\mathrm{SiO}_{2}\right.$, 9:1 $\mathrm{CH}_{2} \mathrm{Cl}_{2}$-ethyl acetate): ${ }^{1} \mathrm{H} \mathrm{NMR}\left(400 \mathrm{MHz}, \mathrm{CDCl}_{3}\right) \delta 7.74(\mathrm{~d}, \mathrm{~J}=8.6 \mathrm{~Hz}, 2 \mathrm{H}), 7.35-7.32(\mathrm{~m}$, 4H), 7.01-6.99 (m, 4H), 6.87 (d, J = 8.6 Hz, 4H), 6.78 (d, J = 8.6 Hz, 2H), 6.70 (d, J = 8.6 Hz, 2H), 5.31 (s, 2H), 4.55 (s, br, 2H), 4.30 (q, J = 7.1 Hz, 2H), 1.33 (t, J = 7.1 Hz, 3H); ${ }^{13} \mathrm{C}$ NMR $\left(100 \mathrm{MHz}, \mathrm{CDCl}_{3}\right) \delta 166.22,154.44,146.86,143.23,131.19,130.29,129.94,129.66,128.90$, 128.06, 125.88, 125.09, 124.87, 123.47, 115.23, 61.05, 47.18, 14.24; MS (EI) m/z: $575\left(\mathrm{M}^{+}\right)$.

G1-ester 11. Diphenol 10i (0.56 g; $0.97 \mathrm{mmol})$, alcohol 3i (0.75 g; $2.1 \mathrm{mmol})$ and $\mathrm{PPh}_{3}(0.77 \mathrm{~g}$; $2.9 \mathrm{mmol})$ were dissolved in dry THF $(10 \mathrm{ml})$. DEAD (0.51 g; $2.9 \mathrm{mmol})$ was added and the mixture was stirred at room temperature during $5 \mathrm{~h}$ under argon atmosphere. The solvent was 
evaporated and G1-ester 11 was obtained after column chromatography $\left(\mathrm{SiO}_{2}, 2: 1 \mathrm{CH}_{2} \mathrm{Cl}_{2}\right.$ petroleumether) as an amorphous solid (0.56 g; 47\%): ${ }^{1} \mathrm{H}$ NMR (400 MHz, $\left.\mathrm{CDCl}_{3}\right) \delta 7.74$ (d, J $=8.5 \mathrm{~Hz}, 2 \mathrm{H}), 7.38(\mathrm{~d}, \mathrm{~J}=8.5 \mathrm{~Hz}, 4 \mathrm{H}), 7.35-7.33(\mathrm{~m}, 12 \mathrm{H}), 7.25-7.23(\mathrm{~d}, \mathrm{~J}=8.5 \mathrm{~Hz}, 4 \mathrm{H}), 7.00-$ 6.98 (m, 12H), $6.90(\mathrm{~d}, \mathrm{~J}=8.5 \mathrm{~Hz}, 4 \mathrm{H}), 6.90$ (s, 4H), 6.84 (d, J = 8.5 Hz, 4H), 6.77 (d, J = 8.5 Hz, 2H), 5.33 (s, 6H), 4.97 (s, 4H), 4.27 (q, J = $7 \mathrm{~Hz}, 2 \mathrm{H}), 1.29$ (t, J = $7 \mathrm{~Hz}, 3 \mathrm{H}) ;{ }^{13} \mathrm{C}$ NMR (100 $\left.\mathrm{MHz} \mathrm{CDCl}_{3}\right) \delta 157.49,146.74,146.59,133.07,133.03,130.92,129.65,128.87,128.83,125.10$, 125.05, 124.86, 123.47, 123.39, 119.96, 114.48, 111.08, 69.57, 47.21, 47.02, 14.23; MS (ES) $\mathrm{m} / \mathrm{z}: 1255.6\left(\mathrm{MNH}_{4}^{+}\right), 1238.6\left(\mathrm{MH}^{+}\right)$.

G1-alcohol 12. G1-ester 11 (0.50 g; $0.40 \mathrm{mmol})$ was dissolved in dry THF (5 ml) and added dropwise to a suspension of $\mathrm{LiAlH}_{4}(32 \mathrm{mg} ; 84 \mathrm{mmol})$ in dry THF (5 ml). After complete addition the suspension was refluxed for $10 \mathrm{~min}$. After cooling to room temperature a solution of $\mathrm{NaOH}$ (1M; app. $5 \mathrm{ml}$ ) was added dropwise and the solution was filtered and the precipitate washed with THF ( 2 x $5 \mathrm{ml}$ ). After column chromatography $\left(\mathrm{SiO}_{2}, \mathrm{CH}_{2} \mathrm{Cl}_{2}\right)$ the alcohol 12 was obtained as an amorphous solid (0.44 g; 92\%): ${ }^{1} \mathrm{H}$ NMR (400 MHz, $\left.\mathrm{CDCl}_{3}\right) \delta 7.35$ (d, J = 8.5 Hz, 4H), 7.35-7.31 (m 12H), 7.22 (d, J = 8.5 Hz, 4H), 7.01 (d, J = 8.5 Hz, 2H), 6.99-6.96 (m, 12H), 6.91 (d, J = 8.5 Hz, 4H), 6.88 (s, 4H), 6.81 (d, J = 8.5 Hz, 4H), 6.72 (d, J = 8.5 Hz, 2H), 5.34 (s, 2H), 5.33 (s, 4H), 4.96 (s, 4H), 4.48 (s, 2H); ${ }^{13} \mathrm{C}$ NMR (100 MHz, $\left.\mathrm{CDCl}_{3}\right) \delta 157.23$, 146.96, 146.57, 140.89, 138.74, 138.27, 133.12, 133.02, 130.85, 129.58, 129.16, 128.84, 127.40, 126.68, 125.93, 125.28, 125.10, 124.93, 123.38, 119.90, 114.37, 111.05, 69.52, 64.62, 47.19.

G1-bromoacetate 13. G1-alcohol 12 (3.6 g; $3.0 \mathrm{mmol})$ and $\mathrm{Et}_{3} \mathrm{~N}$ (0.76 g; $7.5 \mathrm{mmol}$ ) were dissolved in $\mathrm{CH}_{2} \mathrm{Cl}_{2}(10 \mathrm{ml})$ and the solution was placed under argon and cooled to $0^{\circ} \mathrm{C}$. Via a septum, bromoacetyl bromide was added $(1.4 \mathrm{~g} ; 7.0 \mathrm{mmol})$ and the mixture was stirred at room temperature during $30 \mathrm{~min}$. The solvent was evaporated and after column chromatography $\left(\mathrm{SiO}_{2}\right.$, $\mathrm{CH}_{2} \mathrm{Cl}_{2}$ ), the bromide 13 was obtained as an amorphous solid (3.3 g; 83\%): ${ }^{1} \mathrm{H} \mathrm{NMR}(250 \mathrm{MHz}$, $\left.\mathrm{CDCl}_{3}\right) \delta$ 7.50-7.46 (m, 16H), 7.17 (AA'BB', 8H), 6.99 (d, J = 8.5 Hz, 2H), 6.98-6.95 (m, 12H), $6.92(\mathrm{~d}, \mathrm{~J}=8.5 \mathrm{~Hz}, 4 \mathrm{H}), 6.85$ (s, 4H), 6.82 (d, J = $8.5 \mathrm{~Hz}, 4 \mathrm{H}), 6.72$ (d, J = $8.5 \mathrm{~Hz}, 2 \mathrm{H}), 5.36$ (s, 2H), 5.31 (s, 4H), 4.99 (s, 2H), 4.89 (s, 4H), 3.67 (s, 2H); $\left.{ }^{13} \mathrm{C} \mathrm{NMR} \mathrm{(62.5} \mathrm{MHz,} \mathrm{CDCl}_{3}\right) \delta$ 157.27, 146.81, 146.50, 140.79, 139.08, 132.96, 132.88, 130.80, 129.72, 129.21, 128.97, 128.75, 128.16, 127.94, 125.84, 125.04, 124.92, 123.33, 119.80, 114.36, 110.98, 69.42, 67.00, 47.12, 25.55 .

G1-dendrimer 14. G1-bromoacetate 13 (0.18 g; $0.14 \mathrm{mmol})$, DPP 6 (18 mg; $0.064 \mathrm{mmol}$ ), $\mathrm{K}_{2} \mathrm{CO}_{3}$ (36 mg; $0.25 \mathrm{mmol}$ ) were suspended in DMF (15 ml) and heated under argon atmosphere during $48 \mathrm{~h}$. The reaction mixture was evaporated and after column chromatography $\left(\mathrm{SiO}_{2}\right.$, $\mathrm{CH}_{2} \mathrm{Cl}_{2}$ ) G1-dendrimer 14 was obtained a yellow glassy solid (0.13 g; 72\%): ${ }^{1} \mathrm{H}$ NMR (400 $\left.\mathrm{MHz} \mathrm{CDCl}_{3}\right) \delta 7.59$ (dd, J = 7.1 Hz, J = 1.2 Hz, 4H), 7.35-7.28 (m, 38H), 7.19 (d, J = 8.5 Hz, 8H), 7.00-6.96 (m, 24H), 6.89 (2 x d, J = 8.6 Hz, 12H), $6.86(\mathrm{~s}, 8 \mathrm{H}), 6.80(\mathrm{~d}, \mathrm{~J}=8 \mathrm{H}), 6.69(\mathrm{~d}, \mathrm{~J}=$ 4H), 5.34 (s, 4H), 5.13 (s, 8H), 4.96 (s, 4H), 4.90 (s, 8H), 4.14 (s, 4H); ${ }^{13} \mathrm{C}$ NMR (100 MHz, $\left.\mathrm{CDCl}_{3}\right) \delta 168.18,162.08,157.34,146.91,146.57,140.88,139.03,133.08,133.01,131.55$, 130.84, 129.75, 128.90, 128.54, 127.83, 127.31, 125.89, 125.09, 124.98, 123.38, 119.87, 114.38, 111.04, 109.62, 69.49, 47.190, 47.189, 43.48; MS (ES) $2761.9\left(\mathrm{MH}^{+}\right)$.

G1-dendrimer 15. This dendrimer was obtained analogously to but with use of 1,3,5-tris(4hydroxyphenyl)benzene in refluxing acetone. This procedure afforded 15 in 65\% yield as a white glassy solid: ${ }^{1} \mathrm{H}$ NMR (250 MHz, $\mathrm{CDCl}_{3}$ ) $\delta 7.51$ (s, 3H), 7.47 (d, J = 8.5 Hz, 6H), 7.34-7.28 (m, 48H), 7.16 (d, J = 8.5 Hz, 12H), 7.04 (d, J = 8.5 Hz, 6H), 7.00-6.93 (m, 54H), 6.91 (d, J = 8.5 
Hz, 12H), 6.83 (s, 12H), 6.82 (d, J = 8.5 Hz, 12H), 6.77 (d, J = 8.5 Hz, 6H), 5.34 (s, 6H), 5.29 (s, 12H), 5.11 (s, 6H), 4.89 (s, 12H, 4.56 (s, 6H); MS (ES) m/z $2032\left(\mathrm{MH}_{2}\right)^{2+}$.

Ethyl ester 10h. Diels-Alder adduct 9b (5.5 g; $13 \mathrm{mmol}$ ) and ethyl 4-aminophenyl acetate (2.84 g; $16 \mathrm{mmol})$ and p-TsOH (0.5 g) were dissolved in m-xylene (40 ml) and the mixture was heated at reflux temperature in a Dean-Start apparatus during $24 \mathrm{~h}$ under argon atmosphere. After cooling to room temperature and evaporation of the solvent, methanol (20 ml) was added. After filtration and washing with methanol (3 $55 \mathrm{ml}$ ), pyrroloanthracene 10h was obtained as a white solid (4.65 g; 63\%): ${ }^{1} \mathrm{H}$ NMR (250 MHz, $\left.\mathrm{CDCl}_{3}\right) \delta$ 7.39-7.36 (m, 4H), 7.24-7.20 (m, 6H), 7.037.00 (m, 10H), 6.73 (d, J = 8 Hz, 2H), 5.41 (s, 2H), 4.12 (q, J = 7 Hz, 2H), 3.48 (s, 2H), 1.28 (t, J $=7 \mathrm{~Hz}, 3 \mathrm{H})$; MS (EI) m/z $557\left(\mathrm{M}^{+}\right)$.

Alcohol 10k. The ester 10h (4.6 g; $8.3 \mathrm{mmol}$ ) was dissolved in dry THF (25 ml) and added in a dropwise manner to a suspension of $\mathrm{LiAlH}_{4}(0.61 \mathrm{~g} ; 16 \mathrm{mmol})$ in dry THF $(5 \mathrm{ml})$. After complete addition, the mixture was refluxed for $1 \mathrm{~h}$. After analogous work-up as before and column chromatography ( $\mathrm{SiO}_{2}, 20: 1 \mathrm{CH}_{2} \mathrm{Cl}_{2}$-ethyl acetate), alcohol 10k was obtained as a foaming thick oil (4.0 g; $93 \%$ ): ${ }^{1} \mathrm{H}$ NMR (250 MHz, $\left.\mathrm{CDCl}_{3}\right) \delta$ 7.38-7.32 (m, 4H), 7.23-7.19 (m, $6 \mathrm{H}), 7.02-6.98$ (m, 8H), 6.91 (d, J = 8.5 Hz, 2H), 6.71 (d, J = 8.5 Hz, 2H), 5.40 (s, 2H), 3.71 (t, J $=7 \mathrm{~Hz}, 2 \mathrm{H}), 2.73(\mathrm{t}, \mathrm{J}=7 \mathrm{~Hz}, 2 \mathrm{H}) ; \mathrm{MS}(\mathrm{EI}) \mathrm{m} / \mathrm{z}: 515\left(\mathrm{M}^{+}\right)$.

Mesylate 10l. The alcohol 10k (0.50 g; $0.97 \mathrm{mmol}), \mathrm{Et}_{3} \mathrm{~N}(0.60 \mathrm{~g} ; 6.0 \mathrm{mmol})$ and DMAP (0.050 g) were dissolved in $\mathrm{CH}_{2} \mathrm{Cl}_{2}(20 \mathrm{ml})$ and the solution was cooled to $0^{\circ} \mathrm{C}$ under argon atmosphere. Mesyl chloride (0.55g; $4.9 \mathrm{mmol}$ ) was added and the mixture was stirred at room temperature during $30 \mathrm{~min}$. After evaporation of the solvent and column chromatography $\left(\mathrm{SiO}_{2} ; \mathrm{CH}_{2} \mathrm{Cl}_{2}\right)$ the mesylate 101 was obtained as a yellowish oil (0.53 g; 93\%): ${ }^{1} \mathrm{H} \mathrm{NMR}\left(250 \mathrm{MHz}, \mathrm{CDCl}_{3}\right) \delta 7.38-$ 7.32 (m, 4H), 7.23-7.19 (m, 6H), 7.02-6.98 (m, 8H), 6.91 (d, J = 8.5 Hz, 2H), 6.74 (d, J = 8.5 Hz, 2H), 5.41 (s, 2H), 4.27 (t, J = $7 \mathrm{~Hz}, 2 \mathrm{H}), 2.87$ (t, J = $7 \mathrm{~Hz}, 2 \mathrm{H}), 2.63$ (s, 3H); ${ }^{3} \mathrm{C}$ NMR (62.5 $\left.\mathrm{MHz} \mathrm{CDCl}_{3}\right) \delta 146.76,137.85,134.63,132.15,130.35,129.59,129.35,128.86,127.92,126.68$, 126.23, 124,95, 123.42, 69.88, 47.05, 37.08, 34.86; MS (CI) m/z: $594\left(\mathrm{MH}^{+}\right)$.

G0-dendrimer 17. The mesylate 101 ( $0.35 \mathrm{~g}$; $0.69 \mathrm{mmol})$, 1,3,5-tris(4-hydroxyphenyl)benzene (74 mg; $0.21 \mathrm{mmol}), \mathrm{K}_{2} \mathrm{CO}_{3}(0.14 \mathrm{~g} ; 1.0 \mathrm{mmol})$ and 18-crown-6 (25 mg) were dissolved in acetone $(15 \mathrm{ml})$ and refluxed under argon atmosphere during $48 \mathrm{~h}$. After evaporation and column chromatography $\left(\mathrm{SiO}_{2}, 2: 1 \mathrm{CH}_{2} \mathrm{Cl}_{2}\right.$-petroleum ether) dendrimer 17 was obtained as a white solid (0.14 g; 35\%): ${ }^{1} \mathrm{H}$ NMR (250 MHz, $\mathrm{CDCl}_{3}$ ) $\delta 7.62$ (s, 3H), 7.57 (d, J = 8.5 Hz, 6H), 7.42-7.34 (m, 12H), 7.23-7.17 (m, 18H), 7.06-6.98 (m, 24H), 6.92 (d, J = 8.5 Hz, 6H), 6.73 (d, J = 8.5 Hz, 6H), 5.42 (s, 6H), 4.13 (t, J = $7 \mathrm{~Hz}, 6 \mathrm{H}), 3.03$ (t, J = $7 \mathrm{~Hz}, 6 \mathrm{H})$.

Ethyl ester 10g. Diels-Alder adduct $9 b(0.50 \mathrm{~g} ; 1.1 \mathrm{mmol})$, ethyl 4-aminophenyl acetate (0.25 g; $1.4 \mathrm{mmol}$ ) and $\mathrm{p}$-TsOH (50 mg) were dissolved in m-xylene (20 ml) and the solution was refluxed during $48 \mathrm{~h}$ under argon atmosphere under azeotropic removal of water in a Dean-Start tube. After cooling down and evaporation, methanol $(10 \mathrm{ml})$ was added. Compound $\mathbf{1 0 g}$ was obtained after filtration and washing with methanol (2 x $5 \mathrm{ml}) \quad(0.58 \mathrm{~g} ; 85 \%):{ }^{1} \mathrm{H}$ NMR (250 $\left.\mathrm{MHz} \mathrm{CDCl}_{3}\right) \delta$ 7.37-7.33 (m, 4H), 7.00-6.97 (m, 6H), $6.93(\mathrm{~d}, \mathrm{~J}=8.5 \mathrm{~Hz}, 4 \mathrm{H}), 6.76(\mathrm{~d}, \mathrm{~J}=8.5$ Hz, 4H), 6.73 (d, J = 8.5 Hz, 2H), 5.38 (s, 2H), 4.09 (q, J = 7 Hz, 2H), 3.76 (s, 6H), 3.48 (s, 2H), 1.18 (t, J = $7 \mathrm{~Hz}, 3 \mathrm{H}) ;{ }^{13} \mathrm{C}$ NMR $\left(100 \mathrm{MHz}, \mathrm{CDCl}_{3}\right) \delta 171.19,158.00,146.98,137.87,130.78$, 129.40, 129.14, 129.08, 125.90, 124.90, 123.36, 113.40, 60.69, 55.06, 47.13, 40.87, 14.06; MS (EI) $\mathrm{m} / \mathrm{z}: 617\left(\mathrm{M}^{+}\right)$. 
Diphenol 10j. Dimethoxy derivative $10 \mathrm{~g}(0.36 \mathrm{~g} ; 0.58 \mathrm{mmol})$ was dissolved in dichloromethane (20 ml) and the solution was cooled to $-78^{\circ} \mathrm{C}$ under argon atmosphere. Via a septum, $\mathrm{BBr}_{3}(1.46$ $\mathrm{ml}(1 \mathrm{M}) ; 1.5 \mathrm{mmol})$ was added and the mixture was stirred at $-20^{\circ} \mathrm{C}$ during $15 \mathrm{~h}$. Water $(5 \mathrm{ml})$ and ethyl acetate $(20 \mathrm{ml})$ were added and the mixture was extracted once more with ethyl acetate ( $10 \mathrm{ml})$. The diphenol $\mathbf{1 0 j}$ was obtained as a light brown powder $(0.27 \mathrm{~g} ; 75 \%$ ) after column chromatography $\left(\mathrm{SiO}_{2}, 9: 1 \mathrm{CH}_{2} \mathrm{Cl}_{2}\right.$-ethyl acetate): ${ }^{1} \mathrm{H}$ NMR $\left(250 \mathrm{MHz}, \mathrm{CDCl}_{3}\right) \delta$ 7.35-7.32 (m, 4H), 6.98-6.92 (m, 6H), 6.86 (d, J = 8.5 Hz, 4H), 6.70-6.65 (m, 6H), 5.91 (s, 2H), 5.34 (s, 2H), 4.12 (q, J = $7 \mathrm{~Hz}, 2 \mathrm{H}$ ), 3.48 (s, 2H), 1.26 (t, J = $7 \mathrm{~Hz}, 3 \mathrm{H})$; MS (ES) m/z $589\left(\mathrm{M}^{+}\right)$.

G1-ester 19. The alcohol 10k (1.0 g; $1.9 \mathrm{mmol}), \mathrm{AB}_{2}$ monomer $10 \mathrm{j}(0.52 \mathrm{~g} ; 0.88 \mathrm{mmol})$ and $\mathrm{PPh}_{3}$ (0.69 g; $\left.2.6 \mathrm{mmol}\right)$ were dissolved in dry THF (10 ml). To this solution, DEAD (0.46 g; 2.6 mmol) was added and the mixture was stirred during $3 \mathrm{~h}$ under argon atmosphere. After evaporation and column chromatography $\left(\mathrm{SiO}_{2}, 2: 1 \mathrm{CH}_{2} \mathrm{Cl}_{2}\right.$-petroleum ether), G1-ester 19 was obtained as a white solid (1.0 g; 71\%): ${ }^{1} \mathrm{H}$ NMR $\left(400 \mathrm{MHz}, \mathrm{CDCl}_{3}\right) \delta$ 7.38-7.36 (m, 8H), 7.347.32 (m, 4H), 7.22-7.18 (m, 8H), 7.03-6.99 (m, 20H), 6.89 (d, J = 8.5 Hz, 4H), 6.74-6.70 (m, 10H), 5.41 (s, 4H), 5.32 (s, 2H), 4.08-4.03 (m, 6H), 3.44 (s, 2H), 2.97 (t, J = $7 \mathrm{~Hz}, 4 \mathrm{H}), 1.13$ (t, J $=7 \mathrm{~Hz}, 3 \mathrm{H}) ;{ }^{13} \mathrm{H}$ NMR $\left(100 \mathrm{MHz}, \mathrm{CDCl}_{3}\right) \delta 171.19,157.24,147.01,146.95,137.90,137.32$, 136.57, 132.35, 132.23, 130.79, 130.29, 129.68, 129.48, 129.11, 128.87, 127.92, 126.66, 126.16, 125.93, 124.99, 124.93, 123.48, 123.40, 114.13, 68.35, 60.71, 47.22, 40.86, 35.26, 14.08; MS (ES) $\mathrm{m} / \mathrm{z}: 1586\left(\mathrm{MH}^{+}\right)$.

\section{Acknowledgements}

We thank the KULeuven and the FWO Vlaanderen for their continuing support. M.S. thanks the FWO Vlaanderen for a postdoctoral fellowship.

\section{References}

1. For recent reviews see for example Grayson, S.M.; Fréchet, J.M.J. Chem. Rev. 2001, 101, 3819. Dendrimers and Dendrons: Concepts, Syntheses, Applications; Newkome, G.R.; Moorefield, C.N.; F. Vögtle, $2^{\text {nd }}$ Ed.,Wiley-VCH 2001, Weinheim, New York.

2. (a) Newkome, G.R.; Moorefield, C.N.; Baker, G.R.; Johnson, A.L.; Behera, R.K. Angew. Chem. Int. Ed. Engl. 1991, 30, 1176. (b) Newkome, G.R.; Moorefield, C.N.; Baker, G.R.; Saunders, M.J.; Grossman, S.H. Angew. Chem. Int. Ed. Engl. 1991, 30, 1178. (c) Jansen, J.F.G.A.; de Brabander-van den Berg, E.M.M.; Meijer, E.W. Science 1994, 266, 1226. (d) de Brabander-van den Berg, E.M.M.; Jansen, J.F.G.A.; Meijer, E.W. J. Am. Chem. Soc. 1995, 117, 4417. (e) de Brabander-van den Berg, E.M.M.; Jansen, J.F.G.A.; Meijer, E.W. Stevelmans, S., van Boxtel, D.A.F.J., van Hest, J.C.M. J. Am. Chem. Soc. 1996, 118, 7398.

3. Shaklai, K.; Hart, H. J. Org. Chem. 1991, 56, 6905.

4. Kearney, P.; Mizoue, L.; Kumpf, R., Forman, A.; McCurdy, A.; Dougherty, D.A. J. Am. Chem. Soc. 1993, 115, 9907.

5. (a) Weber, E.; Finge, S.; Reutel, C. J. Prakt. Chem. 1993, 335, 147. (b) Weber, E.; Finge, S.; Csöregh, I. J. Org. Chem. 1991, 56, 7281.

6. Jye-Shane, Y.; Swager, M.T. J. Am. Chem. Soc. 1998, 120, 11864. 
7. Smet, M.; Corens, D.; Van Meervelt, L.; Dehaen, W. Molecules 2000, 5, 179.

8. Elming, N.; Clauson-Kaas, N. Acta Chem. Scand. 1952, 6, 535.

9. Iqbal, A.; Jost, M., Kirchmayr, R.; Pfenninger, J.; Rochat, A.; Wallquist, O. Bull. Soc. Chim. Belg. 1988, 97, 615.

10. (a) Hurd, C.D.; Tockman, A. J. Am. Chem. Soc. 1959, 81, 116. (b) Parakka, J.P.; Sadanandan, E.V.; Cava, M.P. J. Org. Chem. 1994, 59, 3014.

11. Mitsunobu, O.; Yamada, M.; Mukaiyama, T. Bull. Chem. Soc. Jap. 1967, 40, 935. 